\title{
Novel Solvent Systems for Cellulose Dissolution
}

\begin{abstract}
Jianbo Shuai and Xiaohui Wang*
Cellulose, as the most abundant sustainable resource on earth, can be chemically transformed into a variety of biodegradable materials, which have been proposed as the ideal substitutes for plastic products. The first challenge for the fabrication of cellulose-based functional materials is the successful dissolution of cellulose by solvents. However, most existing cellulose solvents have environmental, economic, and other drawbacks that limit their further industrial applications. Research on developing novel solvent systems with "greener" and "cheaper" properties is needed to meet the challenges.
\end{abstract}

Keywords: Cellulose dissolution; Solvent systems; Ionic liquids; Deep eutectic solvents

Contact information: State Key Laboratory of Pulp and Paper Engineering, South China University of Technology, Guangzhou, 510640, P. R. China. E-mail:fewangxh@scut.edu.cn

\section{The Urgent Need for Novel Solvent Systems}

With increasing concerns related to non-degradable plastic waste, the development of environmentally friendly materials from renewable biomass-based resources is becoming increasingly attractive. Cellulose, as the most abundant and biodegradable polymer, has been considered as an ideal material from which to prepare substitutes for petroleum-based plastics. Once dissolved, cellulose can be fabricated into multiple materials, such as cellulose fibers, films, hydrogels, aerogels, and composite materials, which show great potential for applications in textiles, packaging, biomedical materials, etc. (Tu et al. 2020). However, because of its strong intermolecular and intramolecular network of hydrogen bonding, cellulose neither can be melted nor dissolved in most used solvents, and this has limited its prospects for many applications.

There are a number of classical solvent systems for cellulose dissolution, such as $\mathrm{NaOH} / \mathrm{CS}_{2}$ (viscose process), N-methylmorpholine-N-oxide (NMMO, Lyocell process), $\mathrm{LiCl} / N, N$-dimethylacetamide (DMAc), tetrabutylammonium fluoride/dimethyl sulfoxide (TBAF/DMSO), and $\mathrm{NaOH} /$ urea, etc. These solvent systems have greatly promoted the development of the cellulose chemical industry, although most of them suffer from various drawbacks, such as instability, high toxicity, high cost, poor recyclability, harsh processing conditions, and low solubility capability. For instance, in the viscose rayon procedure, the use of the volatile and highly toxic $\mathrm{CS}_{2}$ may cause serious environmental problems. The Lyocell process relying on NMMO solvent may trigger oxidative side reactions that lead to difficult recovery and uncontrolled explosive reactions (Kuzmina et al. 2017). Therefore, an ongoing search for environmentally friendly solvent systems that can be efficiently recycled remains a key focus in terms of cellulose dissolving and processing. 


\section{Ionic Liquids (ILs) for Cellulose Dissolution}

Various novel "green" solvent systems recently have become available for cellulose dissolution. Among them, ILs as novel cellulose solvents have attracted considerable attention as competitive candidates for industrial applications due to their attractive properties, such as high thermal tolerance, chemical stability, and excellent dissolution ability (Rieland et al. 2020). ILs, which consist combinations of different cations and anions, were first reported as cellulose solvents by Swatloski et al. (2002). These novel solvents for cellulose dissolution can mainly be divided into 3 groups: heterocyclic amines (imidazolium, pyridinium, etc.), quaternary cations (ammonium- and phosphonium-based cores), and superbases (DBNH, DBUH, etc.).

Imidazolium-based ionic liquids are the most used ILs to dissolve cellulose for regenerating cellulose-based functional materials. For example, Zhang et al. (2005) found that 1-allyl-3-methylimidazolium chloride $(\mathrm{AmimCl})$ is a powerful solvent for cellulose dissolution. After coagulating of cellulose/ILs solutions into water, regenerated cellulose fibers or transparent films can be obtained. Superbase-derived ionic liquids as relatively newly discovered ILs, have received increasing attention due to their favorable combination of low cost and high ability to dissolve cellulose. Sixta and coworkers (2015) focused on a superbase-derived IL of 1,5-diazabicyclo [4.3.0] non-5-enium acetate ([DBNH][OAc]), which allowed the dissolution of cellulose and regeneration of the cellulose as highly oriented fibres (called Ioncell-F), which have tenacity values higher than those of commercial rayon and Lyocell fibers.

Although there are many advantages of ILs as cellulose solvents, their high cost, high viscosity, and relatively high toxicity may restrict their further practical applications. Improving the recyclability of ILs after cellulose processing is necessary to decrease the production costs as well as the environmental impacts of ILs. The regenerating of cellulose solution is usually processed with a large amount of water, which inevitably leads to large volumes of low-concentration IL wastewater (less than $5 \mathrm{wt} \%$ ). The most promising method for separating ILs is to remove water from such waste aqueous solution. Several commonly used methods for recycling ILs from waste aqueous solution have been evaluated, such as liquid-liquid extraction, adsorption, distillation, electrodialysis, crystallization, phase separation, and membrane-related methods (Sklavounos et al. 2016). However, the concentration after recycling using most methods is not high enough (lower than $90 \mathrm{wt} \%$ ) to meet the requirements for further reusage. Although high concentrations can be obtained by distillation-based methods, the process tends to require high energy input. Moreover, most of the studies conducted on recycling process of ILs have been at laboratory scale, and there has not been enough validation from industrial practice. The recycling technologies still need to be improved for large-scale industrial production.

\section{Deep Eutectic Solvents (DESs) for Cellulose Dissolution}

The preparation of DESs involves mixing a hydrogen bond acceptor (HBA) and a hydrogen bond donor (HBD). The strong ability of the DES to undergo hydrogen bond interactions means that the mixture will have a much lower melting point than the starting components. Compared with traditional solvents, DESs possess several superior properties, including easiness in preparation, low cost, and relatively low toxicity (Wang et al. 2020). The DESs can interact effectively with carbohydrates, thus leading to their dissolution. Compared with ILs, DESs are more suitable as solvents for pretreatment processes than as cellulose solvents owing to the relatively poor capability to dissolve cellulose. On the other 
hand, the study about cellulose dissolution and recycling process in DESs is still in its early stages. DES systems suitable for cellulose processing need to be further explored and optimized.

\section{Future Opportunities}

As novel solvent systems of cellulose, ILs and DESs have attracted considerable attention as potential replacements for traditional solvents. However, the problems of costs, toxicity, and recycling technology need to be well addressed before their further utilization, in particular for the large-scale industrial applications. The following several directions should be points for future research and development of solvent systems for cellulose dissolution.

(I) Design and synthesis of new "greener" and "cheaper" cellulose solvents with strong dissolving capability, stability, and ease of recycling should be a continuing focus.

(II) Research is already under way to optimize the existing ILs and DESs systems. For instance, the viscosity, dissolution temperature, and energy consumption can be decreased by mixing ILs/ DESs with different co-solvents.

(III) Attention is needed to develop recycling techniques for various solvent systems to decrease their cost in terms of energy and to increase their sustainability. Integrated recycling procedure combining different recycling technologies should be considered, because a single method can hardly address most problems.

(IV) The research of dissolving systems should be performed in parallel with the studies on cellulose materials.

\section{Acknowledgements}

The authors would like to acknowledge support from the National Key Research and Development Program of China (2019YFE0114400) and the Taishan Industrial Leading Talent Project (20180215).

\section{References Cited}

Kuzmina, O., Bhardwaj, J., Vincent, S. R., Wanasekara, N. D., Kalossaka, L. M., Griffith, J., Potthast, A., Rahatekar, S., Eichhorn, S. J., and Welton, T. (2017). "Superbase ionic liquids for effective cellulose processing from dissolution to carbonization," Green Chemistry 19, 5949-5957. DOI: 10.1039/C7GC02671D

Rieland, J. M., and Love, B. J. (2020). "Ionic liquids: A milestone on the pathway to greener recycling of cellulose from biomass," Resources, Conservation and Recycling 155, 104678. DOI: 10.1016/j.resconrec.2019.104678

Sixta, H., Michud, A., Hauru, L., Asaadi, S., Ma, Y., King, A. W. T., Kilpeläinen, I., and Hummel, M. (2015). "Ioncell-F: A high-strength regenerated cellulose fibre," Nordic Pulp \& Paper Research Journal 30, 43-57. DOI: 10.3183/npprj-2015-30-01-p043057

Sklavounos, E., Helminen, J. K., Kyllönen, L., Kilpeläinen, I., and King, A. W. (2016). "Ionic liquids: recycling," Encyclopedia of Inorganic and Bioinorganic Chemistry. DOI: 10.1002/9781119951438.eibc2451

Swatloski, R. P., Spear, S. K., Holbrey, J. D., and Rogers, R. D. (2002). "Dissolution of cellose with ionic liquids," Journal of the American Chemical Society 124 (18), 49744975. DOI: $10.1021 / \mathrm{ja} 025790 \mathrm{~m}$ 
Tu, H., Zhu, M., Duan, B., and Zhang, L. (2020). "Recent progress in high-strength and robust regenerated cellulose materials," Advance Materials 2000682. DOI: 10.1002/adma.202000682

Wang, C., Yang, S., Song, X., Pi, Q., Zhang, Q., Liu, Q., Xu, Y., Chen, L., and Ma, L. (2020). "Novel solvent systems for biomass fractionation based on hydrogen-bond interaction: A minireview," Advance Sustainable Systems 4, 2000085. DOI: 10.1002/adsu.202000085

Zhang, H., Wu, J., Zhang, J., and He, J. (2005). "1-Allyl-3-methylimidazolium chloride room temperature ionic liquid: A new and powerful nonderivatizing solvent for cellulose," Macromolecules 38, 8272-8277. DOI: 10.1021/ma0505676 